\title{
An Accuracy Assessment Method for Two-Dimensional Functional Data in Simulation-Based Design
}

\author{
Michael J. Alexander ${ }^{*}$ and Panos Y. Papalambros ${ }^{\dagger}$ \\ University of Michigan, Ann Arbor, MI, 48104-2125, USA
}

\begin{abstract}
Accuracy assessment methods are necessary for functional data used in simulation-based design optimization to ensure model and optimal solution validity. Although many error metrics exist and perform well for one-dimensional (1D) applications, the suitability of such metrics for higher dimensional functional data, such as two-dimensional (2D) performance maps, have been largely unexplored. This paper examines the extension of the 1D accuracy assessment method AVASIM to 2D applications, in support of decomposition-based multidisciplinary design optimization (MDO) coordination strategies that require measurement of the consistency of functional data exchanged among subproblems through error metrics. Specifically, AVASIM is used as a consistency measure in the coordination strategy of analytical target cascading that requires functional data consisting of $1 \mathrm{D}$ torque curves and 2D power loss maps from motors to be exchanged among subproblems in electric vehicle design optimization. Results indicate that a generalized AVASIM formulation is an effective consistency measure for accuracy and consistency in a computationally efficient manner.
\end{abstract}

\section{Introduction}

$\mathrm{M}$ any engineering applications utilize computer simulations to model the physical characteristics of new products and systems. Engineers must typically validate these models against some physical, experimental data to ensure that the simulations are capturing the behavior of the system accurately. Among the quantities required for validation are functional data, and their accuracy is usually assessed using some error metric, such as mean-square error (MSE) or root-mean-square error (RMSE). Most of these error metrics have been developed for one-dimensional (1D) functional data and perform well for many applications. However, the performance and suitability of these metrics is largely unexplored for higher dimensional functional data, such as the two-dimensional (2D) performance maps used for engines, motors, and pumps. Inability to assess effectively the accuracy of such representations can lead to erroneous predictions of system performance and poor design decisions. The accuracy assessment problem for higher dimensional functional data is not limited to the validation of simulation models against physical, experimental data; it may also occur in the validation of functional data approximations against previously validated functional data from high-fidelity simulations. One particular area of interest in this latter case is multidisciplinary design optimization (MDO) coordination strategies, such as analytical target cascading (ATC). Effective error metrics are necessary to assess the discrepancy between coupled quantities, including functional data, which are derived from two different sources to facilitate MDO convergence. It is therefore necessary that a suitable error metric be implemented to help engineers select physically realizable optimal designs.

The application domain that motivates the present paper is electric vehicle (EV) powertrain design using ATC. Specifically, approximations of 1D motor torque curves and 2D power loss maps generated by the system level optimization problem must be matched to their high-fidelity versions generated by the subsystem level optimization problem to facilitate ATC convergence. Section II provides a brief background on various 1D error metrics and identifies AVASIM $^{1}$ as a suitable candidate for extension to 2D problems. Section III describes AVASIM in detail, including the adjustments necessary for 2D problems, and demonstrates the algorithm's effectiveness in assessing functional data approximation error offline (i.e., not during optimization). ATC is then reviewed in Section IV along with its problem formulation for EV powertrain design. Section V describes the results for implementations

\footnotetext{
* Graduate Student, Department of Mechanical Engineering, 3200 EECS c/o 2250 G.G. Brown, 2350 Hayward Street, University of Michigan, Ann Arbor, MI 48104-2125.

${ }^{\dagger}$ Donald C. Graham Professor of Engineering, Department of Mechanical Engineering, 3200 EECS c/o 2250 G.G. Brown, 2350 Hayward Street, University of Michigan, Ann Arbor, MI 48104-2125, AIAA Member.
}

1

American Institute of Aeronautics and Astronautics 
using RMSE and AVASIM as measures of consistency between functional data. Finally, Section VI offers conclusions regarding the usefulness of this method and implications for other possible metrics.

\section{Background}

The literature on the accuracy assessment of 1D functional data through error metrics is vast. Sarin provides a comprehensive list and description of these methods, along with key error measures such as magnitude, phase, and shape $^{2}$. Because the current application deals with the validation of functional data approximations against functional data from high-fidelity simulations, the phase error measure is not as significant. Instead, error metrics that are robust and primarily address magnitude and shape error measures are considered here. Vector norms, for example, form the basis of many error metrics, such as MSE and RMSE, and are relatively simple to use ${ }^{2}$. Average residuals and their standard deviations are also straightforward in their implementation, but have the disadvantage of cancellation for comparisons containing positive and negative residuals ${ }^{2}$. The coefficient of correlation and $0^{\text {th }}-2^{\text {nd }}$ order relative difference of moments are slightly more advanced than vector norms and, in the absence of significant phase error, possess effective measures of magnitude error ${ }^{2}$. Sprague and Geers' error metric ${ }^{3-5}$ and the similar Russell's error metric ${ }^{6-7}$ possess a measure that specifically addresses magnitude error; however, neither of these metrics can address shape error. Conversely, the normalized integrated square $\operatorname{error}^{8}$ does possess measures of magnitude and shape error, but cannot account for the shape error in the overall metric. Dynamic time warping ${ }^{9-13}$ and the error assessment of response time histories metric, also known as $\mathrm{EARTH}^{2}$, are advanced methods that effectively measure the magnitude and shape errors, but have the disadvantage of extensive computational time as they both require the solution of a dynamic programming problem. Although any of the aforementioned techniques can be selected in an initial experimentation of accuracy assessment for $2 \mathrm{D}$ functional data, it is preferable to start with the simplest meaningful approach to gain some early understanding. Of particular interest are error metrics that systematically, objectively and efficiently provide a clear indication of local and global functional data accuracy with respect to preset thresholds ${ }^{14}$. With these in mind, the metric selected for an initial study in this work is the Accuracy and Validity Algorithm for SIMulation, or AVASIM ${ }^{1}$.

\section{Accuracy and Validity Algorithm for Simulation}

$\operatorname{AVASIM}^{1}$ is an accuracy assessment tool that characterizes the local and global error between baseline and approximation functional data through $\oint_{1}$-norms and residual sums. Using these measures, error indices are constructed such that nonnegative values denote valid functional data approximations with accuracy levels between 0 and 1 , and all negative values generally denote invalid functional data approximations. Validity is defined by functional data approximations that lie within some predetermined threshold value; therefore, a value of 0 indicates that a functional data approximation is at the threshold and valid, whereas a value of 1 indicates that a functional data approximation is completely accurate.

\section{A. Algorithm}

The algorithm begins by selecting points of interest, known as target points ${ }^{14}$, from the baseline functional data. These target points are used to calculate the local error index between the baseline and approximation functional data through an $\zeta_{1}$-norm indirectly. In addition, a percentage error tolerance $t l_{i}$ must be assigned to each target point based on its desired accuracy level. The local error index between the baseline and approximation functional data for a single target point is therefore

$$
E_{\text {local }, i}=1-\frac{\left|y_{i}-\hat{y}_{i}\right|}{y_{i} \text { tol }_{i}}
$$

where $y_{i}$ and $\hat{y}_{i}$ denote target point values from the baseline and approximation functional data, respectively. Observe that the above formulation degrades when $y_{i} \leq 0$ as this would either lead to division by zero or index values greater than 1 . On a practical level, the division-by-zero issue may be resolved by setting $y_{i}=\delta$, where $\delta$ is a small, positive number. The index value issue can be alleviated by simply taking the absolute value of the denominator in Eq. (1). With these problems addressed, an overall measure of the local error can be found by averaging $E_{\text {local, }}$ for all $n_{p}$ target points: 


$$
E_{\text {local }}=\frac{1}{n_{p}} \sum_{i=1}^{n_{p}} E_{\text {local }, i}
$$

In the next phase, it is necessary to calculate the residual sum between the baseline and approximation functional data $^{14}$. This value is used in conjunction with another residual sum between the baseline functional data and some threshold functional data to compute the global error index. The first residual sum is given by

$$
R S_{a p p}=\int_{0}^{X}|y(x)-\hat{y}(x)| d x
$$

where $y(x)$ and $\hat{y}(x)$ represent the complete baseline and approximation functional data respectively and $X$ is the domain over which the functional data are defined. Similarly, the second residual sum is given by

$$
R S_{\text {thresh }}=\int_{0}^{X}\left|y(x)-y_{\text {thresh }}(x)\right| d x, \quad y_{\text {thresh }}(x)=a y(x+b)
$$

where $y_{\text {thresh }}(x)$ represents the complete threshold functional data. It is this residual sum that sets a maximum acceptable value for the global error. Note that the amplitude threshold coefficient $a$ and the phase threshold coefficient $b$ help set this value based on tol $_{i}$ :

$$
a=1+\min \left(\text { tol }_{i}\right), \quad b=\min \left(\mid \text { tol }_{i} x_{i}^{\text {base }} \mid\right)
$$

With this definition, the global error index between the baseline and approximation functional data is given by

$$
E_{\text {global }}=1-\frac{R S_{a p p}}{R S_{\text {thresh }}}
$$

The combined error index $E_{c o m b}$ is found by simply calculating the arithmetic mean of the results of Eqs. (2) and (6). Typically, $E_{\text {comb }}$ is used to gain a sense not only of the overall error between the functional data but also of whether an approximation is even valid with respect to the preset tolerances. Such a condition is referred to as a liberal validity criterion $^{14}$ as it only requires $E_{\text {comb }}$ to be nonnegative for valid curve approximations. Conversely, if it is required that $E_{\text {local }}$ and $E_{\text {global }}$ (and hence $E_{\text {comb }}$ ) be nonnegative, then this condition is known as a conservative validity criterion ${ }^{14}$.

Based on Eqs. (1)-(6), it is proposed that AVASIM be extended to assess the accuracy of 2D functional data by simply modifying the residual sums with double integration. Let $z(x, y)$ and $z(x, y)$ represent the baseline and approximation functional data, respectively. Then the residual sum between the baseline and approximation functional data is

$$
R S_{a p p}=\iint_{D}|z(x, y)-\hat{z}(x, y)| d x d y
$$

where $D$ is the domain over which the functional data are defined. Likewise, the residual sum between the baseline and threshold functional data is

$$
R S_{\text {thresh }}=\iint_{D}\left|z(x, y)-z_{\text {thresh }}(x, y)\right| d x d y, \quad z_{\text {thresh }}(x, y)=a z(x+b, y+c)
$$

where $a$ is still the amplitude threshold coefficient and $b$ and $c$ are phase threshold coefficients for $x$ and $y$ respectively. Note that $b$ and $c$ are determined by:

$$
b=\min \left(\mid \text { tol }_{i} x_{i}^{\text {base }} \mid\right), \quad c=\min \left(\left|\operatorname{tol}_{i} y_{i}^{\text {base }}\right|\right)
$$


The rest of the algorithm remains the same, including the meaning of the validity criteria.

\section{B. Application: Accuracy Assessment of Motor Performance Curves/Map}

AVASIM was applied to an accuracy assessment problem in which the functional data included maximum and minimum torque curves and a power loss map originally produced by a motor simulation model within an EV powertrain simulation for automobiles. Because these functional data were computationally expensive to use directly in an MDO coordination strategy, approximations were developed offline to improve optimization efficiency. These approximations were therefore validated against their high-fidelity versions at the expected optimal design, with the 1D AVASIM formulation used for the torque curves and the 2D AVASIM formulation used for the power loss map. Since the target points selected in both cases were merely mesh points describing the functional data, a uniform tolerance of $t o l_{i}=0.10$ was assigned for all points. For similar reasons, the phase threshold coefficients were set to zero, as in Sohns, ${ }^{14}$ as opposed to the methodology described in Eqs. (5) and (9). Additionally, division-by-zero errors were avoided by setting $\delta=10^{-4}$ for the torque curves and $\delta=1$ for the power loss map based on experience. Finally, note that the number of target points for the power loss map was not known a priori but rather determined by a subroutine that only included points that were within the torque curve boundaries. A similar subroutine was used to define numerically the domain of integration $D$ for the power loss map, which is also within the torque curve boundaries. The results from AVASIM are shown in Table 1, and Figs. 1-2 illustrate the accuracy of these functional data approximations visually.

Upon reviewing these results, it is evident that AVASIM reasonably describes the local and global accuracy of the functional data approximations. Specifically, it is seen that the conservative validity criterion is satisfied for all approximations and that the combined error indices for the torque curves and power loss map indicate accuracies of $76.5 \%, 96.6 \%$, and $58.4 \%$, respectively. It should be observed that in each case, the global error index is significantly higher than the local error index, which is consistent with what is seen visually. Such behavior is often sufficient and in fact desirable for many engineering applications. Therefore, one can utilize this knowledge and modify the AVASIM formulations such that $E_{\text {comb }}$ is not an arithmetic mean of $E_{\text {local }}$ and $E_{\text {global }}$, but rather a weighted sum,

$$
E_{\text {comb }}=w_{\text {local }} E_{\text {local }}+w_{\text {global }} E_{\text {global }}
$$

where $w_{\text {local }}$ and $w_{\text {global }}$ are nonnegative weights whose sum must always equal 1 . This new formulation is referred to as generalized AVASIM because it enables users to determine which accuracy component is more important when computing the combined error index. Such flexibility could have important implications when implementing AVASIM in an MDO coordination strategy.

Table 1. AVASIM results for functional data approximations

\begin{tabular}{cccc}
\hline Index & Max-Torque & Min-Torque & Power Loss \\
\hline$E_{\text {local }}$ & 0.588 & 0.969 & 0.319 \\
$E_{\text {global }}$ & 0.942 & 0.963 & 0.849 \\
$E_{\text {comb }}$ & 0.765 & 0.966 & 0.584 \\
\hline
\end{tabular}






Figure 1. Torque curve comparison

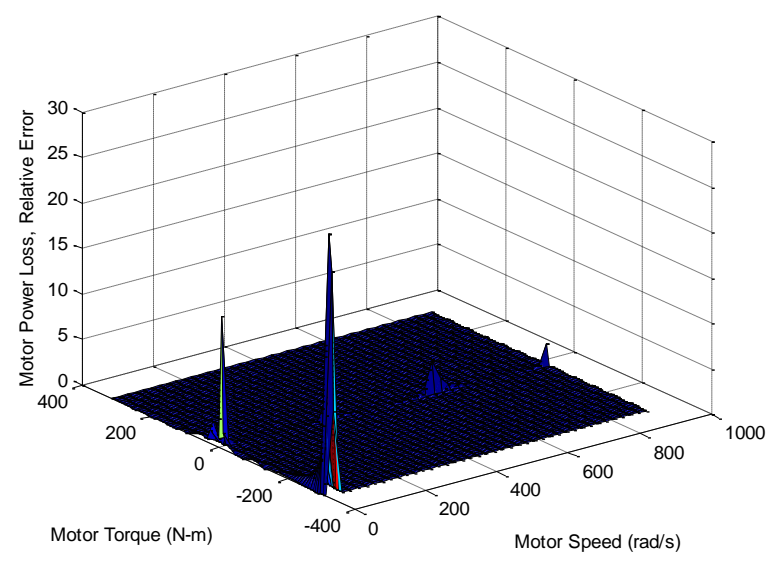

Figure 2. Power loss map relative error

\section{Analytical Target Cascading}

$\mathrm{ATC}^{15-16}$ is an MDO coordination strategy that is used for large-scale, multilevel design problems in which design targets at upper levels are passed, or cascaded, down to lower levels to optimize some local performance objective. However, at the same time, a second objective consisting of a penalty function is minimized with each performance objective to ensure that design targets assigned at the upper levels are realizable by subsystem responses.

\section{A. General Problem Formulation}

It is assumed here that the original design problem is multilevel, or has been decomposed into a multilevel problem consisting of $i$ levels and $j$ elements. Because the decomposition is hierarchical, any subproblem linked above/below subproblem $P_{\mathrm{ij}}$ is known as its parent/child. Using this terminology, the general ATC subproblem formulation for $P_{\mathrm{ij}}$ is defined as ${ }^{15}$ :

$$
\begin{aligned}
& \min _{\overline{\mathbf{x}}_{i j}} f_{i j}\left(\overline{\mathbf{x}}_{i j}\right)+\pi\left(\mathbf{c}\left(\overline{\mathbf{x}}_{11}, \cdots, \overline{\mathbf{x}}_{N M}\right)\right) \\
& \text { subject to } \mathbf{g}_{i j}\left(\overline{\mathbf{x}}_{i j}\right) \leq \mathbf{0}, \mathbf{h}_{i j}\left(\overline{\mathbf{x}}_{i j}\right)=\mathbf{0} \\
& \text { where } \quad \overline{\mathbf{x}}_{i j}=\left[\mathbf{x}_{i j}, \mathbf{r}_{i j}, \mathbf{t}_{(i+1) k_{1}}, \cdots, \mathbf{t}_{(i+1) k_{c i j}}\right], \mathbf{c}=\left[\mathbf{c}_{22}, \cdots, \mathbf{c}_{N M}\right]
\end{aligned}
$$

In the above, $\mathbf{x}_{\mathrm{ij}}$ is the vector of local design variables, $\mathbf{t}_{\mathrm{ij}}$ is the vector of target linking variables passed from the element's parent at level $(i-1), \mathbf{r}_{\mathrm{ij}}$ is the vector of response linking variables passed to the element's parent at level $(i-1), \mathbf{c}_{\mathrm{ij}}=\mathbf{t}_{\mathrm{ij}}-\mathbf{r}_{\mathrm{ij}}$ is the vector of (relaxed) consistency constraints between target and response linking variables, $f_{\mathrm{ij}}$ is the local objective function, $\pi$ is the penalty function, $\mathbf{g}_{\mathrm{ij}}$ is the vector of inequality constraints, $\mathbf{h}_{\mathrm{ij}}$ is the vector of equality constraints, $N$ is the number of levels, and $M$ is the total number of elements. It should be noted that in this study, the linking variables in $\mathbf{t}_{\mathrm{ij}}$ and $\mathbf{r}_{\mathrm{ij}}$ only consist of coupling variables and do not included shared variables. Additionally, the definition for the relaxed consistency constraints is only applicable for scalar-valued linking variables; when these terms consist of functional data, the definition must be modified based on the error metric desired for measuring consistency. For example, in previous work ${ }^{17-19}$, consistency between functional data was measured through RMSE as $c_{i j, l}=\sqrt{\sum_{m=1}^{Q}\left(t_{i j, m}-r_{i j, m}\right)^{2} / Q}$ for the $t^{\text {th }}$-component of $\mathbf{c}_{\mathrm{ij}}$, where $Q$ denotes the number of mesh points defining the functional data. Because consistency in this study will be measured through AVASIM, the definition for the relaxed consistency constraints is $c_{i j, l}=1-E_{c o m b, l}$, where $E_{c o m b, l}$ is computed through Eqs. (1)(9) with $t_{i j, m}$ and $r_{i j, m}$ substituted for $y_{i}$ and $\hat{y}_{i}$ as appropriate. Observe that in both cases, the definition of consistency is similar to that of the scalar-valued case; that is, when $c_{i j, l}=0$, the linking variables match exactly, whereas if $c_{i j, l} \neq$ 0 , there is some discrepancy among the subproblems. However, using AVASIM enhances the meaning of consistency since $c_{i j, l}=1$ indicates that the functional data are consistent and at the threshold, and $c_{i j, l}>1$ indicates 
that the functional data is inconsistent. Finally, note that for the current coordination strategy, the algorithm terminates when $\left\|\mathbf{c}^{(K)}-\mathbf{c}^{(K-I)}\right\|_{\infty}$ is within some small tolerance, where $K$ denotes the iteration number.

Because an augmented-Lagrangian (AL) penalty function was used in this work, the general ATC subproblem formulation was modified accordingly ${ }^{20}$ :

$$
\begin{aligned}
& \min _{\overline{\mathbf{x}}_{i j}} f_{i j}\left(\overline{\mathbf{x}}_{i j}\right)-\mathbf{v}_{i j}^{T} \mathbf{r}_{i j}+\sum_{k \in C_{i j}} \mathbf{v}_{(i+1) k}^{T} \mathbf{t}_{(i+1) k}+\left\|\mathbf{w}_{i j} \circ\left(\mathbf{t}_{i j}-\mathbf{r}_{i j}\right)\right\|_{2}^{2}+\sum_{k \in C_{i j}}\left\|\mathbf{w}_{i j} \circ\left(\mathbf{t}_{(i+1) k}-\mathbf{r}_{(i+1) k}\right)\right\|_{2}^{2} \\
& \text { subject to } \mathbf{g}_{i j}\left(\overline{\mathbf{x}}_{i j}\right) \leq \mathbf{0}, \mathbf{h}_{i j}\left(\overline{\mathbf{x}}_{i j}\right)=\mathbf{0} \\
& \text { where } \quad \overline{\mathbf{x}}_{i j}=\left[\mathbf{x}_{i j}, \mathbf{r}_{i j}, \mathbf{t}_{(i+1) k_{1}}, \cdots, \mathbf{t}_{(i+1) k_{c i j}}\right]
\end{aligned}
$$

Here, the vectors $\mathbf{v}$ and $\mathbf{w}$ are weights corresponding to the linear and quadratic terms in the AL penalty function, respectively. The subproblems are solved in an inner loop strategy where the weights remain constant. Upon inner loop convergence, termination conditions are evaluated in the outer loop and, if another inner loop execution is required, the penalty weights are updated as indicated below:

$$
\begin{gathered}
\mathbf{v}^{(K+1)}=\mathbf{v}^{(K)}+2 \mathbf{w}^{(K)} \circ \mathbf{w}^{(K)} \circ \mathbf{c}^{(K)} \\
\mathbf{w}^{(K+1)}=\beta \mathbf{w}^{(K)}, \text { where } \beta \geq 1
\end{gathered}
$$

The information flow for the general ATC-AL subproblem is illustrated in Fig. 3.

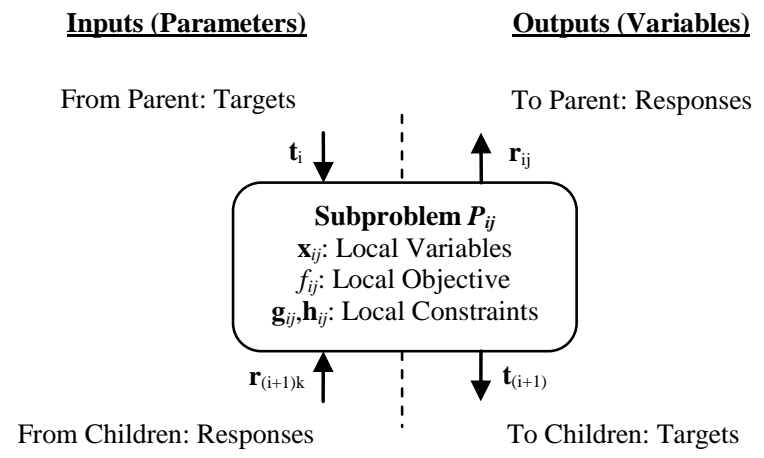

Figure 3. ATC information flow ${ }^{20}$

\section{B. Problem Formulation for EV Powertrain Design}

The design problem formulation for the EV powertrain consists of a bi-level ATC decomposition. In particular, the top level subproblem optimizes the battery design and location, belt-drive ratios, and motor map selection for maximum gasoline-equivalent fuel economy and minimum inconsistency between decision variables coupled with the bottom level subproblem. This problem is also subject to constraints related to packaging, performance, motor feasibility, range, power availability, and battery capacity. The bottom level subproblem, however, optimizes the motor design exclusively for minimum inconsistency between decision variables coupled with the top level subproblem.

Using Eq. (12), the top level subproblem $P_{11}$ (excluding simple bound constraints) is formulated as: 


$$
\begin{aligned}
& \min _{\overline{\mathbf{x}}_{11}}-m p g_{e}\left(\overline{\mathbf{x}}_{11}\right)+\mathbf{v}_{22}^{T}\left(\mathbf{t}_{22}-\mathbf{r}_{22}\right)+\left\|\mathbf{w}_{22} \circ\left(\mathbf{t}_{22}-\mathbf{r}_{22}\right)\right\|_{2}^{2} \\
& \text { subject to } g_{11,1}\left(\overline{\mathbf{x}}_{11}\right)=b_{w}\left(\overline{\mathbf{x}}_{11}\right)-b_{\text {wmax }} \leq 0 \quad g_{11,5}\left(\overline{\mathbf{x}}_{11}\right)=\omega_{V}\left(\overline{\mathbf{x}}_{11}\right) \leq 0
\end{aligned}
$$

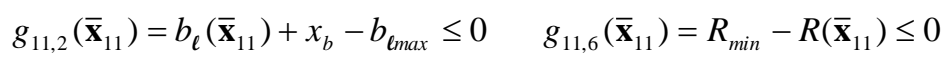

$$
\begin{aligned}
& g_{11,3}\left(\overline{\mathbf{x}}_{11}\right)=t_{60}\left(\overline{\mathbf{x}}_{11}\right)-t_{60 \max } \leq 0 \quad g_{11,7}\left(\overline{\mathbf{x}}_{11}\right)=P_{V}\left(\overline{\mathbf{x}}_{11}\right) \leq 0 \\
& g_{11,4}\left(\overline{\mathbf{x}}_{11}\right)=\tau_{V}\left(\overline{\mathbf{x}}_{11}\right) \leq 0 \quad g_{11,8}\left(\overline{\mathbf{x}}_{11}\right)=C_{b}\left(\overline{\mathbf{x}}_{11}\right)-C_{\text {bmax }}\left(\overline{\mathbf{x}}_{11}\right) \leq 0 \\
& \text { where } \quad \overline{\mathbf{x}}_{11}=\left[B_{I}, B_{W}, B_{L}, x_{b}, p_{r}, \mathbf{p}_{c o m b}^{T}, \omega_{\max }^{T}, m_{m}^{T}, J_{r}^{T}, I_{y, m}^{T}, I_{z m}^{T}, y_{m}^{T}\right] \text {, } \\
& \mathbf{t}_{22}=\left[\boldsymbol{\varphi}_{\text {comb }}^{T}, \omega_{m a x}^{T}, m_{m}^{T}, J_{r}^{T}, I_{y, m}^{T}, I_{z m}^{T}, y_{m}^{T}\right], \boldsymbol{\varphi}_{c o m b}^{T}=f\left(\mathbf{p}_{c o m b}^{T}\right), \\
& \mathbf{r}_{22}=\left[\boldsymbol{\varphi}_{c o m b}^{R}, \omega_{\max }^{R}, m_{m}^{R}, J_{r}^{R}, I_{y, m}^{R}, I_{z, m}^{R}, y_{m}^{R}\right]
\end{aligned}
$$

In the above, $g_{11,1}$ and $g_{11,2}$ are battery packaging constraints, $g_{11,3}$ is a performance ( $0-60$ mph time) constraint, $g_{11,4}$ and $g_{11,5}$ are motor feasibility constraints, $g_{11,6}$ is a vehicle range constraint, $g_{11,7}$ is a power availability constraint, and $g_{11,8}$ is a battery capacity constraint. The vectors $\boldsymbol{\varphi}_{\text {comb }}$ and $\mathbf{p}_{\text {comb }}$ refer to the combined functional data approximations of the torque curves and power loss map as well as their combined representation in parameter space, respectively. Finally, note that the superscripts $T$ and $R$ refer to target and response copies of the same coupling variable, respectively.

Similarly, the bottom level subproblem $P_{22}$ (excluding simple bound constraints) is formulated as:

$$
\begin{aligned}
& \begin{aligned}
\min _{\overline{\mathbf{x}}_{22}} \mathbf{v}_{11}^{T}\left(\mathbf{t}_{11}-\mathbf{r}_{11}\right)+\left\|\mathbf{w}_{11} \circ\left(\mathbf{t}_{11}-\mathbf{r}_{11}\right)\right\|_{2}^{2} \\
\text { where } \overline{\mathbf{x}}_{22}=\left[\mathcal{l}_{s}, r_{m}, n_{c}, R_{r}\right], \mathbf{t}_{11}=\left[\boldsymbol{\varphi}_{c o m b}^{T}, \omega_{\max }^{T}, m_{m}^{T}, J_{r}^{T}, I_{y, m}^{T}, I_{z, m}^{T}, y_{m}^{T}\right], \\
\qquad \mathbf{r}_{11}=\left[\boldsymbol{\varphi}_{c o m b}^{R}, \omega_{\max }^{R}, m_{m}^{R}, J_{r}^{R}, I_{y, m}^{R}, I_{z m}^{R}, y_{m}^{R}\right]=f\left(\overline{\mathbf{x}}_{22}\right)
\end{aligned}
\end{aligned}
$$

Table 2 provides definitions for the remaining input/output quantities of the objective and constraint functions for both subproblems. 
Table 2. Definition of input/output quantities of objective/constraint functions

\begin{tabular}{cc}
\hline Quantity & Definition \\
\hline$B_{I}$ & Battery electrode thickness scale \\
$B_{W}$ & Battery cell width scale \\
$B_{L}$ & Number of cell windings \\
$x_{b}$ & Battery compartment clearance $(\mathrm{m})$ \\
$p_{r}$ & Belt drive ratio \\
$\omega_{\max }$ & Maximum motor speed (rad/s) \\
$m_{m}$ & Motor mass $(\mathrm{kg})$ \\
$J_{r}$ & Rotor moment of inertia $\left(\mathrm{kg}-\mathrm{m}^{2}\right)$ \\
$I_{y, m}$ & Motor pitch inertia $\left(\mathrm{kg}-\mathrm{m}^{2}\right)$ \\
$I_{z, m}$ & Motor yaw inertia $\left(\mathrm{kg}-\mathrm{m}^{2}\right)$ \\
$y_{m}$ & Motor lateral com location $(\mathrm{m})$ \\
$m p g_{e}$ & Gasoline-equivalent fuel economy $(\mathrm{mpg})$ \\
$b_{w}$ & Battery width $(\mathrm{m})$ \\
$b_{\ell}$ & Battery length $(\mathrm{m})$ \\
$t_{60}$ & 0-60 mph time $(\mathrm{s})$ \\
$\tau_{V}$ & Torque violation constraint $(\mathrm{N}-\mathrm{m})$ \\
$\omega_{V}$ & Speed violation constraint $(\mathrm{rad} / \mathrm{s})$ \\
$R$ & Vehicle range $(\mathrm{mi})$ \\
$P_{V}$ & Power violation constraint $(\mathrm{W})$ \\
$C_{b}$ & Battery capacity $(\mathrm{A}-\mathrm{h})$ \\
$l_{s}$ & Motor stack length $(\mathrm{m})$ \\
$r_{m}$ & Rotor radius $(\mathrm{m})$ \\
$R_{r}$ & Rotor resistance $(\Omega)$ \\
$n_{c}$ & Number of turns per stator coil \\
\hline &
\end{tabular}

\section{ATC Optimization with Functional Data}

The ATC problem formulation shown in Eqs. (14)-(15) was solved using three different measures of consistency for the functional data: RMSE, AVASIM, and generalized AVASIM. In particular, the RMSE solution was used as a baseline case to which all other solutions were compared. In each case, the design problem was solved using NOMADm ${ }^{\mathrm{Abr} 07}$, which is a derivative-free software optimization package that is based on mesh-adaptive search algorithms and was developed in MATLAB ${ }^{\circledR}$ environment. The default settings for this optimizer were modified for the $P_{11}$ subproblem such that only a Latin hypercube search was performed and only 1,000 function evaluations were permitted. This was necessary to alleviate computational issues associated with memory availability. However, for the $P_{22}$ subproblem, the default settings were sufficient. In the ATC coordination strategy, the weight update parameter was set to $\beta=2.75$, the initial weight vectors for both subproblems were set to $\mathbf{v}=\mathbf{0}$ and $\mathbf{w}=\mathbf{1}$, and the tolerance on $\left\|\mathbf{c}^{(K)}-\mathbf{c}^{(K-I)}\right\|_{\infty}$ for outer loop convergence was set to $10^{-2}$. Note that this outer loop convergence criterion only applied to the RMSE case; for all other cases, the coordination strategy was repeated until the number of ATC iterations performed was the same as the RMSE case. Such a modification was necessary for an equitable comparison of the optimization results. All computational work was performed on a $3 \mathrm{GHz}, 4 \mathrm{MB}$ RAM, Intel ${ }^{\circledR}$ Core $^{\mathrm{TM}} 2$ Duo CPU.

\section{A. RMSE in ATC}

The ATC optimization results using RMSE as a consistency measure for the functional data are shown in Tables 3-5. To avoid ill-performance of this measure due to the vast difference in magnitudes between the torque curves and power loss maps, the RMSE definition was slightly modified for the power loss maps such that baseline values larger than $1 \mathrm{~W}$ in magnitude were used to normalize the errors before being squared. Convergence was achieved after 28 ATC iterations with a runtime of approximately 21.3 hours and resulted in a system solution that was reasonably consistent between both subproblems. The active constraints included the bound constraint on $\omega_{\max }^{T}$ and the battery capacity constraint $\left(g_{11,8}\right)$ in the $P_{11}$ subproblem, which were limited to $755 \mathrm{rad} / \mathrm{s}$ and $200 \mathrm{Ah}$, respectively. Observe that the optimal values of $\mathbf{p}_{\text {comb }}$ are not included here as they are too numerous and not physically meaningful; however, the optimal motor map that they produce (and as stored in $\varphi_{\text {comb }}$ ) is illustrated in 
Fig. 4. The total mass of the vehicle was $1330 \mathrm{~kg}$, with approximately $12 \%(158 \mathrm{~kg})$ of the mass associated with the battery pack. With such a design, the EV could achieve a gasoline-equivalent fuel economy of $143 \mathrm{mpg}$, a 0-60 mph time of $9.56 \mathrm{~s}$, and a range of 105 miles.

Table 3. Optimal decision vector for $\boldsymbol{P}_{11}$ subproblem, RMSE solution

\begin{tabular}{ccccccccccc}
\hline$B_{I}$ & $B_{W}$ & $B_{L}$ & $x_{b}$ & $p_{r}$ & $\omega_{\max }{ }^{T}$ & $m_{m}{ }^{T}$ & $J_{r}{ }^{T}$ & $I_{y, m}{ }^{T}$ & $I_{z, m}{ }^{T}$ & $y_{m}{ }^{T}$ \\
\hline 0.74 & 1.43 & 19.75 & 0.25 & 3.93 & 755 & 148 & 0.28 & 4.11 & 4.41 & 0.34 \\
\hline
\end{tabular}

Table 4. Optimal decision vector $\boldsymbol{P}_{22}$ subproblem, RMSE solution

\begin{tabular}{cccc}
\hline$C_{s}$ & $r_{m}$ & $n_{c}$ & $R_{r}$ \\
\hline 0.09 & 0.12 & 17.90 & 0.13 \\
\hline
\end{tabular}

Table 5. Optimal consistency constraint vector/penalty weights, RMSE solution

\begin{tabular}{cccc}
\hline Consistency Constraint & $\mathrm{c}_{\mathrm{opt}}$ & $\mathrm{v}_{\mathrm{opt}}$ & $\mathrm{W}_{\mathrm{opt}}$ \\
\hline$c_{\varphi, \max }$ & 0.88 & $1.43 \times 10^{23}$ & $7.28 \times 10^{11}$ \\
$c_{\varphi, \min }$ & 0.91 & $1.47 \times 10^{23}$ & $7.28 \times 10^{11}$ \\
$c_{\varphi, \text { pLoss }}$ & 0.47 & $7.90 \times 10^{22}$ & $7.28 \times 10^{11}$ \\
$c_{\text {omax }}$ & 0 & 0 & $7.28 \times 10^{11}$ \\
$c_{\operatorname{mm}}$ & 0.58 & $9.33 \times 10^{22}$ & $7.28 \times 10^{11}$ \\
$c_{J r}$ & 0 & $-3.89 \times 10^{20}$ & $7.28 \times 10^{11}$ \\
$c_{I y, m}$ & -0.04 & $-5.76 \times 10^{21}$ & $7.28 \times 10^{11}$ \\
$c_{I, m}$ & 0.03 & $4.81 \times 10^{21}$ & $7.28 \times 10^{11}$ \\
$c_{y m}$ & 0 & $-2.48 \times 10^{20}$ & $7.28 \times 10^{11}$ \\
\hline
\end{tabular}

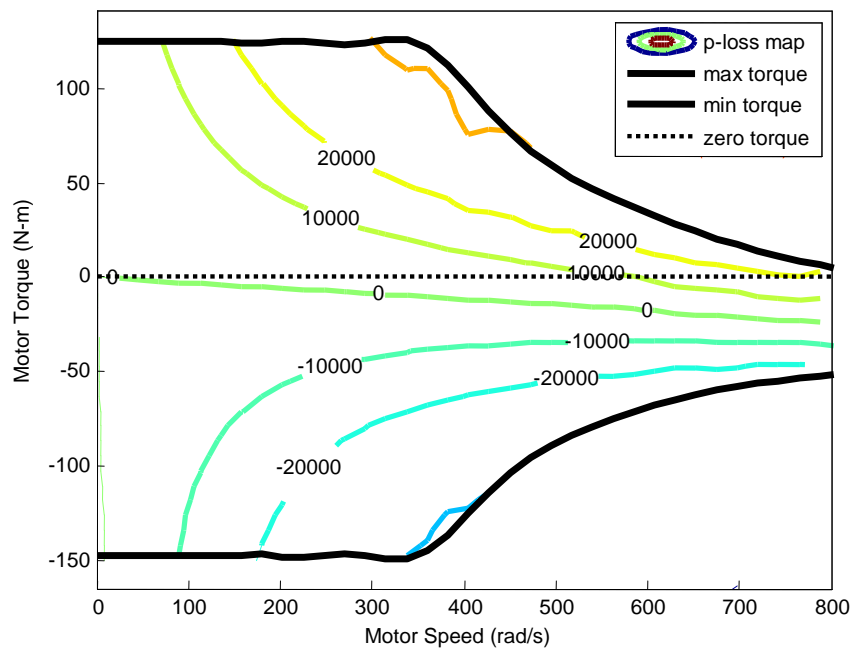

Figure 4. Optimal motor map, RMSE solution

\section{B. AVASIM in ATC}

Similarly, the ATC optimization results using AVASIM as a consistency measure for the functional data are shown in Tables 6-8 and Fig. 5. As indicated earlier, the coordination strategy was repeated until the number of ATC iterations performed was identical to that of the RMSE case. The runtime for this case was 17.8 hours but resulted in a system solution that was inconsistent between the subproblems with respect to the functional data. Therefore, the remaining optimization results have not been interpreted as they lack meaning in the absence of consistency. 
Table 6. Optimal decision vector for $\boldsymbol{P}_{11}$ subproblem, AVASIM solution

\begin{tabular}{ccccccccccc}
\hline$B_{I}$ & $B_{W}$ & $B_{L}$ & $x_{b}$ & $p_{r}$ & $\omega_{\max }{ }^{T}$ & $m_{m}{ }^{T}$ & $J_{r}{ }^{T}$ & $I_{y, m}{ }^{T}$ & $I_{z, m}{ }^{T}$ & $y_{m}{ }^{T}$ \\
\hline 0.74 & 1.43 & 18.15 & 0.25 & 3.73 & 755 & 148 & 0.20 & 2.91 & 4.81 & 0.31 \\
\hline
\end{tabular}

Table 7. Optimal decision vector $\boldsymbol{P}_{22}$ subproblem, AVASIM solution

\begin{tabular}{cccc}
\hline$l_{s}$ & $r_{m}$ & $n_{c}$ & $R_{r}$ \\
\hline 0.13 & 0.11 & 18.13 & 0.05 \\
\hline
\end{tabular}

Table 8. Optimal consistency constraint vector/penalty weights, AVASIM solution

\begin{tabular}{cccc}
\hline Consistency Constraint & $\mathrm{c}_{\mathrm{opt}}$ & $\mathrm{v}_{\mathrm{opt}}$ & $\mathrm{w}_{\mathrm{opt}}$ \\
\hline$c_{\varphi, \max }$ & 2.80 & $4.51 \times 10^{23}$ & $7.28 \times 10^{11}$ \\
$c_{\varphi, \min }$ & 0.86 & $1.39 \times 10^{23}$ & $7.28 \times 10^{11}$ \\
$c_{\varphi, p L o s s}$ & 0.59 & $9.56 \times 10^{22}$ & $7.28 \times 10^{11}$ \\
$c_{\text {omax }}$ & 0 & 0 & $7.28 \times 10^{11}$ \\
$c_{\operatorname{mm}}$ & 0.41 & $6.59 \times 10^{22}$ & $7.28 \times 10^{11}$ \\
$c_{J r}$ & 0 & $-6.79 \times 10^{20}$ & $7.28 \times 10^{11}$ \\
$c_{I y, m}$ & -0.16 & $-2.57 \times 10^{22}$ & $7.28 \times 10^{11}$ \\
$c_{I, m}$ & 0.15 & $2.36 \times 10^{22}$ & $7.28 \times 10^{11}$ \\
$c_{y m}$ & 0.01 & $1.09 \times 10^{21}$ & $7.28 \times 10^{11}$ \\
\hline
\end{tabular}

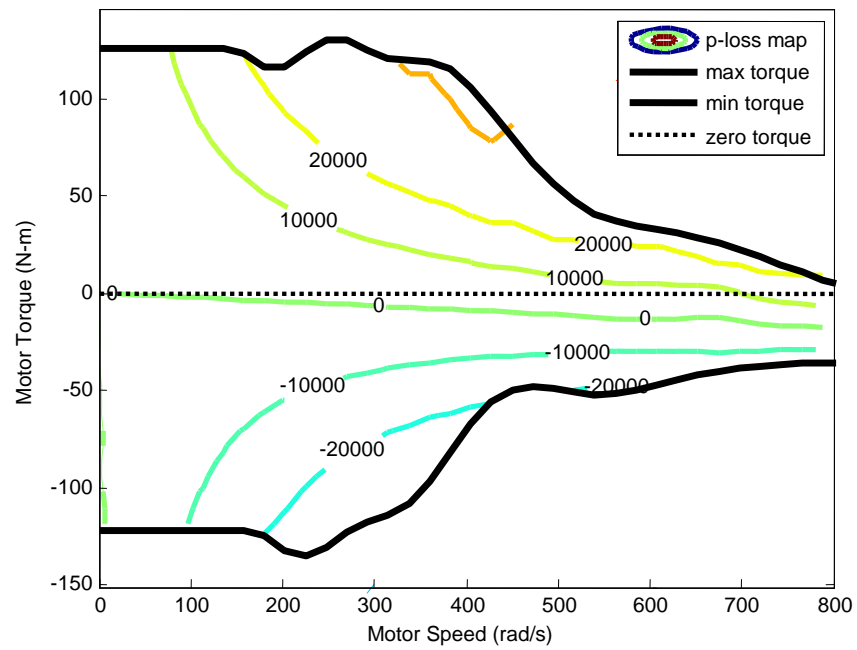

Fig. 5 Optimal motor map, AVASIM solution

\section{Generalized AVASIM in ATC}

Finally, the ATC optimization results using generalized AVASIM as a consistency measure for the functional data are shown in Tables 9-11. The previous results from the optimization strategy using AVASIM appeared to indicate that the global accuracy of the functional data was vital for achieving consistency; therefore, for generalized AVASIM the weights were set to $w_{\text {local }}=1 / 3$ and $w_{\text {global }}=2 / 3$, respectively. As indicated earlier, the coordination strategy was repeated until the number of ATC iterations performed was identical to that of the RMSE case. The runtime for this case was approximately 18.3 hours and resulted in a system solution that was reasonably consistent between both subproblems. In the $P_{11}$ subproblem, the active constraints included the bound constraint on $\omega_{\max }^{T}$, the performance (0-60 mph time) constraint $\left(g_{11,3}\right)$, and the battery capacity constraint $\left(g_{11,8}\right)$, which were limited to 755 
$\mathrm{rad} / \mathrm{s}, 10 \mathrm{~s}$, and $200 \mathrm{Ah}$, respectively. The only activity in the $P_{22}$ subproblem was the bound constraint on $R_{r}$, which was limited to $0.20 \Omega$. The optimal motor map produced by $\mathbf{p}_{\text {comb }}$ is illustrated in Fig. 6 . The total mass of the vehicle was $1330 \mathrm{~kg}$, with approximately $12 \%(158 \mathrm{~kg})$ of the mass associated with the battery pack. With such a design, the EV could achieve a gasoline-equivalent fuel economy of $149 \mathrm{mpg}$ and a range of 109 miles.

Table 9. Optimal decision vector for $\boldsymbol{P}_{11}$ subproblem, generalized AVASIM solution

\begin{tabular}{ccccccccccc}
\hline$B_{I}$ & $B_{W}$ & $B_{L}$ & $x_{b}$ & $p_{r}$ & $\omega_{\max }{ }^{T}$ & $m_{m}{ }^{T}$ & $J_{r}{ }^{T}$ & $I_{y, m}{ }^{T}$ & $I_{z, m}{ }^{T}$ & $y_{m}{ }^{T}$ \\
\hline 0.74 & 1.43 & 19.75 & 0.25 & 3.73 & 755 & 148 & 0.28 & 4.11 & 4.41 & 0.34 \\
\hline
\end{tabular}

Table 10. Optimal decision vector $\boldsymbol{P}_{22}$ subproblem, generalized AVASIM solution

\begin{tabular}{cccc}
\hline$l_{s}$ & $r_{m}$ & $n_{c}$ & $R_{r}$ \\
\hline 0.10 & 0.12 & 17.65 & 0.20 \\
\hline
\end{tabular}

Table 11. Optimal consistency constraint vector/penalty weights, generalized AVASIM solution

\begin{tabular}{cccc}
\hline Consistency Constraint & $\mathrm{c}_{\mathrm{opt}}$ & $\mathrm{v}_{\mathrm{opt}}$ & $\mathrm{W}_{\mathrm{opt}}$ \\
\hline$c_{\varphi, \max }$ & 0.11 & $1.74 \times 10^{22}$ & $7.28 \times 10^{11}$ \\
$c_{\varphi, \min }$ & 0.05 & $8.27 \times 10^{21}$ & $7.28 \times 10^{11}$ \\
$c_{\varphi, p L o s s}$ & 0.36 & $5.88 \times 10^{22}$ & $7.28 \times 10^{11}$ \\
$c_{\text {omax }}$ & 0 & 0 & $7.28 \times 10^{11}$ \\
$c_{m m}$ & 0 & $-5.09 \times 10^{18}$ & $7.28 \times 10^{11}$ \\
$c_{J r}$ & 0 & $3.01 \times 10^{18}$ & $7.28 \times 10^{11}$ \\
$c_{I y, m}$ & 0 & $-1.06 \times 10^{18}$ & $7.28 \times 10^{11}$ \\
$c_{I z, m}$ & 0 & $1.80 \times 10^{18}$ & $7.28 \times 10^{11}$ \\
$c_{y m}$ & 0 & $4.04 \times 10^{18}$ & $7.28 \times 10^{11}$ \\
\hline
\end{tabular}

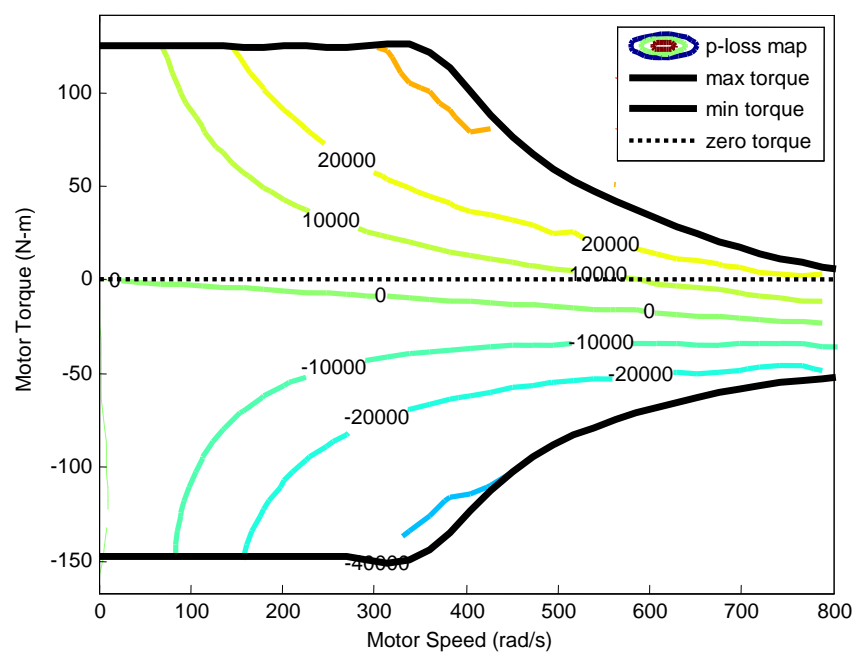

Fig. 6 Optimal motor map, generalized AVASIM solution

\section{Summary of Results}

Initial comparisons among the ATC solutions seem to indicate that the RMSE consistency measure outperformed the AVASIM consistency measure in terms of accuracy, while the generalized AVASIM consistency measure outperformed the RMSE consistency measure in terms of accuracy and efficiency. Indeed, the magnitude of the consistency constraint values for all coupling variables (with the exception of the functional data, since different consistency measures were used) in the RMSE case were generally smaller than those in the AVASIM case, and the optimal motor map appeared to be more accurate in the former case as well. However, the magnitude

11

American Institute of Aeronautics and Astronautics 
of the consistency constraint values for the same coupling variables were smaller in the generalized AVASIM case than those in the RMSE case, and the optimal motor map also appeared to be more accurate in the former case. Moreover, the optimization runtime for the generalized AVASIM case was significantly faster than that of the RMSE case. These results alone could imply that the generalized AVASIM consistency measure is superior in this study. Nevertheless, to alleviate any ambiguity, all ATC solutions were subsequently compared to the solution of the corresponding all-in-one (AiO) MDO problem. Specifically, $\complement_{2}$-norms were used to assess the error between the optimal design vectors of the AiO and ATC solutions, and the percent differences between their associated optima were also calculated. It was found that the error in the optimizers for the RMSE, AVASIM, and generalized AVASIM cases were $0.576,1.74$, and 0.333 , respectively, and that the percent differences in their associated optima were $-9.2 \%, 8.5 \%$, and $-5.3 \%$, respectively. Therefore, these secondary results along with the original observations strongly indicate that the generalized AVASIM consistency measure is superior in this study.

\section{Conclusions and Future Work}

Based on the results in Sections III and V, it is evident that AVASIM can be an effective accuracy assessment tool for both 1D and 2D functional data used in simulation-based design. In both sections, AVASIM was able to provide a clear indication of the accuracy of the functional data with respect to preset error thresholds using relatively simple error measures. Such definitive success indicates the possibility of AVASIM being applicable to more general $n$-dimensional functional data. The nominal AVASIM formulation, which assigns equal weight to both local and global accuracy, was reasonably effective in offline (i.e., not during optimization) accuracy assessment as this was not an iterative procedure over a multitude of possible designs. However, the generalized AVASIM formulation, which enables variable weighting of local and global accuracy according to the problem application, was much more effective during optimization as it interacted with the consistency constraint definitions to provide an optimal solution that was more accurate than the existing RMSE consistency measure. Unlike the latter measure, the generalized AVASIM consistency measure can provide meaningful information regarding the consistency of functional data in an MDO coordination strategy. For example, in Table 11, the generalized AVASIM metric indicates that the consistencies of the maximum/minimum torque curves and the power loss map between the subproblems are $(1-0.11) \times 100 \%=89 \%,(1-0.05) \times 100 \%=95 \%$, and $(1-0.36) \times 100 \%=64 \%$, respectively. The RMSE values in Table 5 for the same functional data are incapable of providing such information. Finally, as an additional benefit, implementing the generalized AVASIM consistency measure resulted in a faster ATC solution time.

Despite the success of the generalized AVASIM metric in the particular problem presented here, several aspects should be investigated in future work. We should determine if a systematic approach can be devised to assign values to $w_{\text {local }}$ and $w_{\text {global }}$. This clearly had a significant impact on the accuracy of the ATC solution, and it is unknown whether a different weighting could further improve the optimization strategy. While it was sufficient in this study to rely on extensive experience with the model and design problem to set values for these weights, such an approach may not be appropriate or efficient in more general problem applications. Further, we should determine the precise reason for the faster convergence times using generalized AVASIM compared to RMSE. This behavior could be more than problem-specific and could yield significant computational savings when using MDO coordination strategies such as ATC. Finally, we should investigate other error metrics and determine their ability to support MDO coordination strategies through the accuracy assessment of 1D and 2D functional data. Following further studies, it may turn out that the generalized AVASIM formulation may not always be the most suitable metric for all applications. However, the present work is a first effort to explore the capabilities of any functional data error metric within an MDO coordination strategy.

\section{Acknowledgments}

This research has been partially supported by the Automotive Research Center, a US Army RDECOM Center of Excellence headquartered at the University of Michigan. This support is gratefully acknowledged.

\section{References}

\footnotetext{
${ }^{1}$ Sendur, P., Stein, J. L., Loucas, L., and Peng, H., “A Model Accuracy and Validity Algorithm”, Proceedings of the ASME International Mechanical Engineering Congress and Exposition, 2002.

${ }^{2}$ Sarin, H., "Error Assessment of Response Time Histories (EARTH): A Metric to Validate Simulation Models", M.S. Thesis, Dept. of Mechanical Engineering, University of Michigan, Ann Arbor, MI, 2008.

${ }^{3}$ Geers, T. L., "Objective Error Measure for the Comparison of Calculated and Measured Transient Response Histories", Shock and Vibration Bulletin, Jun. 1984, pp. 99-107.
} 
${ }^{4}$ Sprague, M. A., and Geers, T. L., "A Spectral-Element Method for Modeling Cavitation in Transient Fluid Structure Interaction", International Journal for Numerical Methods in Engineering, Vol. 60, No. 15, 2004, pp. 2467-2499.

${ }^{5}$ Schwer, L. E., "Validation Metrics for Response Histories: A Review with Case Studies", Technical Report, Schwer Engineering \& Consulting Service, Windsor, CA, 2005.

${ }^{6}$ Russell, D. M., "Error Measures for Comparing Transient Data: Part I, Development of a Comprehensive Error Measure", Proceedings of the $68^{\text {th }}$ Shock and Vibration Symposium, 1997.

${ }^{7}$ Russell, D. M., "Error Measures for Comparing Transient Data: Part II, Error Measures Case Study", Proceedings of the $68^{\text {th }}$ Shock and Vibration Symposium, 1997.

${ }^{8}$ Jacob, C., Charras, F., Trosseille, X., Hamon, J., Pajon, M., and Lecoz, J. Y., "Mathematical Models Integral Rating", International Journal of Crashworthiness, Vol. 5, No. 4, 2000, pp. 417-431.

${ }^{9}$ Keogh, E., and Pazzani, M., "Scaling Up Dynamic Time Warping to Massive Datasets", Proceedings of the $3^{\text {rd }}$ European Conference on Principles and Practice of Knowledge Discovery in Databases, 1999, pp. 1-11.

${ }^{10}$ Ratanamahatana, C. A., and Keogh, E., "Everything You Know about Dynamic Time Warping is Wrong", Proceedings of the $3^{\text {rd }}$ Workshop on Mining Temporal and Sequential Data, in conjunction with the $10^{\text {th }}$ ACM SIGKDD International Conference on Knowledge Discovery and Data Mining, 2004, pp. 22-25.

${ }^{11}$ Liu, J., Cheng, Q., Zheng, Z., and Qian, M., "A DTW-based Probability Model for Speaker Feature Analysis and Data Mining”, Pattern Recognition Letters, Vol. 23, 2002, pp. 1271-1276.

${ }^{12}$ Faundez-Zanuy, M., “On-line Signature Recognition based on VQ-DTW”, Pattern Recognition Letters, Vol. 40, 2007, pp. 981-992.

${ }^{13}$ Fang, P., Wu, Z., Shen, F., Ge, Y., and Fang, B., "Improved DTW Algorithm for Online Signature Verification based on Writing Forces", Proceedings of the International Conference on Intelligent Computing, 2005, pp. 631-640.

${ }^{14}$ Sohns, B., Allison, J., Fathy, H., and Stein, J. L., "Parameterization of a HMMWV Model through the Use of Model Validation Techniques", Proceedings of the Society of Automotive Engineers World Congress, 2006.

${ }^{15}$ Kim, H. M., "Target Cascading in Optimal System Design", Ph.D. Dissertation, Dept. of Mechanical Engineering, University of Michigan, Ann Arbor, MI, 2001.

${ }^{16}$ Kim, H. M., Michelena, N. M., Papalambros, P. Y., and Jiang, T., “Target Cascading in Optimal System Design”, Journal of Mechanical Design, Vol. 125, No. 3, 2003, pp. 474-480.

${ }^{17}$ Alexander, M. J., “Analytical Target Cascading Optimization of an Electric Vehicle Powertrain System”, M.S. Thesis, Dept. of Mechanical Engineering, University of Michigan, Ann Arbor, MI, 2008.

${ }^{18}$ Alexander, M. J., Allison, J. T., and Papalambros, P. Y., "Reduced Representations of Vector-Valued Coupling Variables in Decomposition-based Design Optimization", Proceedings of the $8^{\text {th }}$ World Congress on Structural and Multidisciplinary Optimization, 2009.

${ }^{19}$ Alexander, M. J., Allison, J. T., Papalambros, P. Y., and Gorsich, D. J., "Constraint Management of Reduced Representation Variables in Decomposition-based Design Optimization", Proceedings of the ASME International Design Engineering Technical Conferences \& Computers and Information in Engineering Conference, 2010.

${ }^{20}$ Tosserams, S., Etman, L. F. P., Papalambros, P. Y., and Rooda, J. E., "An Augmented-Lagrangian Relaxation for Analytical Target Cascading using the Alternating Direction Method of Multipliers", Structural and Multidisciplinary Optimization, Vol. 31, 2006, pp. 176-189. 